\title{
Revisión bibliográfica y análisis sobre b-learning y la socialización del alumnado en educación primaria
}

\author{
Literature review and analysis on B-learning and the socialization of students in primary \\ education
}

Fco Esteban Román Sánchez; franest@alumni.uv.es

Diana Marín Suelves; diana.marin@uv.es

José Peirats Chacón; jose.peirats@uv.es

Universitat de Valencia (España)

\section{Resumen}

La modalidad educativa de b-learning se incorporó al sistema educativo hace algunos años y se ha implantado con fuerza, sobre todo a nivel universitario. El objetivo del estudio es analizar las investigaciones publicadas sobre b-learning en la etapa de Educación Primaria a través de una revisión bibliográfica centrada en los últimos 5 años. Aunque el estudio confirma que la tendencia no ha cambiado y el modelo sigue teniendo más presencia en niveles superiores, poco a poco, se van viendo estudios sobre b-learning en Educación Primaria. En su mayoría, son estudios enfocados a la eficacia en cuanto al rendimiento académico, pero podemos encontrar investigaciones donde el foco de estudio se centra en otras aportaciones del modelo. Dentro de la modalidad mixta, el modelo más utilizado y popularizado es el aula invertida, modelo que asume principios pedagógicos tales como el aprendizaje activo, significativo, social, cooperativo... En estos tiempos de pandemia, donde se necesita la distancia social, el reto al que se enfrenta este modelo supone suplir debidamente los beneficios que conlleva la interacción social con propuestas innovadoras e interesantes para el proceso de enseñanza y aprendizaje.

Palabras clave: b-learning, educación primaria, metodología, socialización, intervención

\begin{abstract}
The educational modality of b-learning was incorporated into the educational system a few years ago and has been strongly implemented, especially at the university level. The objective of the study is to analyze the published research on blearning in the Primary Education stage through a Bibliographic Review focused on the last 5 years. Although the study confirms that the trend has not changed and the model continues to have more presence at higher levels, little by little, studies on $b$-learning in primary education are being seen. Most of them are studies focused on effectiveness in terms of academic performance, but we can find research where the focus of study is focused on other contributions of the model. Within the mixedmode, the model most widely used and popularized is the classroom inverted, a model that assumes pedagogical principles such as active learning, significant, social, cooperative... In these times of pandemic, where the social distance, the challenge of this model is to supply adequately the benefits of social interaction with innovative and interesting for the teaching and learning process.
\end{abstract}

Keywords: b-learning, primary education, methodology, socialization, intervention 


\section{INTRODUCCIÓN}

El concepto blended desde su aparición ha tenido diferentes denominaciones: aprendizaje híbrido, semi-presencial, mixto y mezclado (Bartolomé, 2004; Llorente, 2008; Moran, 2012; Picciano, 2014). También visiones o significados distintos, Tayebinik y Puteh (2012) señalan los de blended-learning (b-learning) como combinación de aprendizaje presencial-online; blearning como combinación de sistemas de distribución o tecnologías de distribución de formación y b-learning como combinación de estrategias o modelos de aprendizaje. La primera de las tendencias se ha consolidado y es ampliamente aceptada, en cambio la terminología empleada comúnmente sigue siendo difusa y encontramos términos tales como: híbrido, mixto y blended de manera indistinta. En este estudio nos centraremos en la visión de blended como la combinación de aprendizaje presencial-online. Tradicionalmente, presencial y a distancia han sido los dos grandes sistemas formativos opuestos, pero en este nuevo contexto pandémico están obligados a entenderse.

A continuación, en la Figura 1 se puede ver las publicaciones realizadas sobre Educación Primaria y b-learning en uno de los buscadores más populares y accesibles de divulgación científica, como es DIALNET. Aparte de mostrar la escasez de publicaciones, 52 en los últimos 10 años, lo más significativo de los datos es que ha habido más publicaciones en el año 2020 que durante los años 2000-2009. Estos datos pueden presagiar un aumento creciente de las publicaciones relativas a b-learning en los próximos años, dado su alto interés tras la pandemia. Cabe señalar que los filtros de búsqueda fueron: artículos de revista; ciencias sociales; educación; educación b-learning; educación primaria.

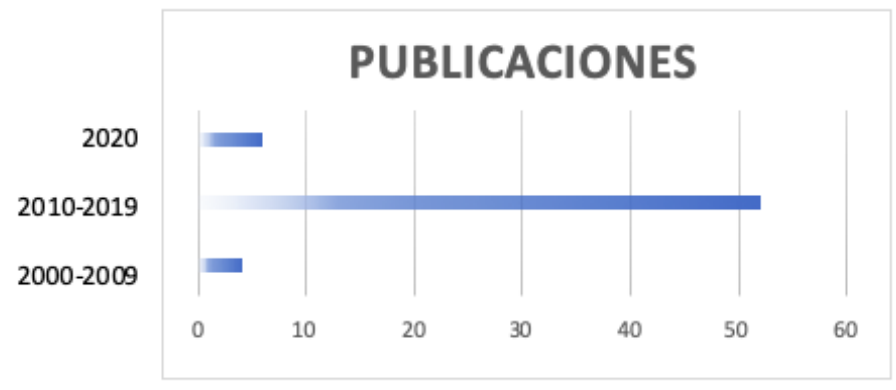

Figura 1. Publicaciones b-learning en Educación Primaria (Dialnet). Fuente: Elaboración propia

Aunque no son muy numerosos los estudios e investigaciones sobre b-learning en Educación Primaria, la revisión que se ofrece en estas páginas pretende averiguar y valorar cómo se han desarrollado dichas investigaciones y qué impacto puede tener en el proceso de enseñanza y aprendizaje. En esa etapa educativa, para el alumnado es importante valorar a los compañeros y compañeras de aula como parte fundamental en su formación y el profesorado tiene que aprovechar el tiempo de clase para provocar contextos donde se relacionen unos con otros. La tecnología puede ser un buen aliado en este proceso; aunque, mucho se ha escrito sobre este aspecto, depende del uso que se haga de ella. Aparte de facilitar la gestión y control en los aprendizajes, se debe centrar su implantación en la creación de contextos y experiencias donde se generen relaciones que ayuden a la socialización y desarrollo personal de todos y todas (Román et al., 2017). Teniendo en cuenta estas palabras, es imprescindible concebir la tecnología como algo más que un medio para la comunicación y el aprendizaje de contenidos. Sin embargo, sea cual sea la postura asumida ante el proceso de digitalización escolar, se debe 
seguir creando y facilitando ambientes de aprendizaje ricos con tareas y dinámicas cooperativas, significativas, constructivas, socializadoras...

La enseñanza semipresencial o b-learning requiere que el docente planifique y desarrolle procesos educativos en los que se superponen tiempo y tareas que acontecen bien en el aula física bien en el aula virtual, sin que necesariamente existan incoherencias entre unas y otras. En esta modalidad de enseñanza el profesorado debe elaborar materiales y plantear actividades para que el alumnado las desarrolle autónomamente fuera del contexto de la clase tradicional. Evidentemente, dentro de este modelo coexisten variantes o grados en función del peso temporal y de trabajo distribuido ante situaciones presenciales y virtuales (Adell y Area, 2009)

Al hilo de lo expuesto, Rosado y Bohadona (2007) señalan que los ambientes virtuales, en general, favorecen: el intercambio de ideas entre individuos, aunque éstas sean diferentes; el diálogo crítico; la reflexión del pensamiento individuo; la negociación como mejor medio para el logro de objetivos en común; el desarrollo de la capacidad de sacar conclusiones; el cambio de experiencias sobre problemas trabajados y sobre el modo de abordarlos; la interacción entre sujetos en tiempos y ambientes distintos; y la ejecución de trabajos colectivos. Aunque las interacciones virtuales no sean tan inmediatas como las que acontecen en las clases físicas tradicionales; no obstante, tienen lugar con más profundidad y su diversidad es mayor en lo que se refiere a temas, culturas y experiencias por la flexibilidad de tiempo y espacio.

La educación mixta mediada por tecnología y una metodología adecuada posibilita nuevas formas estructurales de relacionarse, son "conexiones distendidas" creando ambientes virtuales potencialmente enriquecedores. Estos ambientes virtuales son valorados tanto en el campo educativo como en el social-corporativo. En el primero, se puede destacar las contribuciones de los ambientes virtuales al aprendizaje para los procesos de socialización no sólo entre alumnado y profesorado sino también entre el alumnado. Al mismo tiempo, en esta modalidad b-learning, tanto la experimentación como la simulación son fundamentales en el modelo de aprendizaje mixto o de clase invertida, en tanto que favorecen que el alumnado resuelva problemas y pueda aplicar de manera activa el conocimiento que van adquiriendo (Palau et al., 2015).

Dentro del binomio tecnología y educación, ya hace unos años Salinas (1995) hablaba de tres escenarios que venían configurados por la evolución de las redes de telecomunicaciones y de las potencialidades que aportaban a los procesos de formación: aprendizaje en el hogar, aprendizaje en el puesto de trabajo y aprendizaje en un centro de recursos de aprendizaje o centro de recursos multimedia. Estos tres escenarios que, en aquel momento comenzaban a interconectarse, han ido experimentando modificaciones y avances. Quizá uno de los más importantes ha sido la cualidad de la ubicuidad que las tecnologías móviles han aportado y que ha dado lugar a un cuarto escenario. En efecto, los avances tecnológicos han hecho realidad la ubicuidad del acceso a la información y, en consecuencia, a los recursos de aprendizaje. Es decir, el aprendizaje ubicuo, el aprendizaje en cualquier lugar en cualquier momento (Salinas, 2012).

Elliott (1997: 214) y Pérez Gómez (1998: 92) han destacado que los mecanismos de socialización en la escuela se encuentran en el tipo de estructura de tareas que se trabaja en el aula y en la forma que adquiere la estructura de relaciones sociales. De esta manera detectar el flujo de significados e interacciones que tienen lugar en el aula y en la escuela comportará 
vislumbrar qué valores sociales y pautas culturales se reproducen o transforman a través de la intervención educativa. La estructura de tareas y la estructura social de participación actúan como mediadoras entre las intencionalidades y la realización concreta. De ahí que en los entornos no presenciales o mixtos también se deba prestar atención prioritaria al tipo de tareas y la estructura que genera, y al tipo de intercambios e interacciones producidos en ellas.

En base a lo expuesto se refiere, finalmente, que el objetivo de este estudio es analizar investigaciones publicadas en revistas de impacto en los últimos cinco años sobre intervenciones en Educación Primaria utilizando el modelo blended learning y cómo se puede mejorar la socialización del alumnado en estos entornos.

\section{MÉTODOS}

Para este estudio se utilizaron cuatro bases de datos de reconocido prestigio, como son: Redalyc, Educational Resource Information Center (ERIC), Web of Science (WOS) y SCOPUS. La ecuación de búsqueda se formuló en base a los conceptos clave que guían el estudio: "Aprendizaje mixto" y "Educación Primaria". Los términos fueron traducidos al inglés, estableciéndose finalmente la ecuación: "blended learning" AND "elementary education". En las siguientes líneas, se presentan los criterios de inclusión y exclusión aplicados y, en base a los cuales, se seleccionaron los artículos incluidos en este estudio de revisión bibliográfica (Tabla 1).

\section{Tabla 1.}

Criterios de inclusión y exclusión.

\begin{tabular}{|c|c|}
\hline CRITERIOS DE INCLUSIÓN & CRITERIOS DE EXCLUSIÓN \\
\hline $\begin{array}{l}\text { - } \quad \text { Artículos científicos de investigación } \\
\text { publicados en la red. } \\
\text { - Artículos centrados en el estudio de b-learning } \\
\text { en Educación Primaria. } \\
\text { - } \quad \text { Artículos publicados entre el año 2015-2020. } \\
\text { - } \quad \text { Idioma inglés. }\end{array}$ & $\begin{array}{l}\text { - Artículos teóricos o revisiones. } \\
\text { - } \text { Artículos de implementación de b-learning en } \\
\text { - } \quad \text { Artículos repetidos en las diferentes bases de } \\
\text { datos. }\end{array}$ \\
\hline
\end{tabular}

A partir de las palabras clave, mencionadas anteriormente, se realizó una búsqueda en las bases de datos obteniendo un total de 266 artículos de los cuales, atendiendo a los criterios de inclusión y exclusión, se seleccionaron 21 y se desecharon los 245 restantes porque no se ajustaban al objetivo del estudio. Lo enunciado más arriba queda reflejado en la Tabla 2 y 3 que muestra el proceso seguido para la búsqueda y selección de los artículos que finalmente conforman esta revisión bibliográfica.

\section{Tabla 2.}

Temáticas de los artículos b-learning.

\begin{tabular}{|l|l|l|l|l|l|l|}
\hline \multirow{2}{*}{ Buscador } & \multicolumn{6}{|c|}{ Clasificación según temática del artículo } \\
\cline { 2 - 7 } & 1 & 2 & 3 & 4 & 5 & TOTAL \\
\hline
\end{tabular}




\begin{tabular}{|c|c|c|c|c|c|c|}
\hline WOS & 6 & 39 & 13 & 24 & 0 & 82 \\
\hline ERIC & 43 & 3 & 7 & 2 & 18 & 73 \\
\hline Realdyc & 24 & 56 & 15 & 4 & 2 & 101 \\
\hline SCOPUS & 4 & 2 & 2 & 1 & 1 & 10 \\
\hline TOTAL & 76 & 100 & 37 & 31 & 21 & 266 \\
\hline
\end{tabular}

Nota. (1) Revisión/Marco Teórico, (2) Contexto Universitario/EESS, (3) Formación Docente, (4) Plataforma/Programa/Aplicación, (5) Intervención Educativa. Fuente: elaboración propia.

\section{Tabla 3.}

Proceso de búsqueda.

\begin{tabular}{|l|l|l|l|l|}
\hline $\begin{array}{l}\text { CONCEPTO } \\
\text { DE BÚSQUEDA }\end{array}$ & $\begin{array}{l}\text { - Artículos científicos } \\
\text { Publicados en la reducción } \\
\text { Entre 2015-2020 } \\
\text { Idioma: Inglés }\end{array}$ \\
\hline BUSCADOR & WOS & ERIC & \multicolumn{4}{l|}{ Realdyc } & SCOPUS \\
\hline IDENTIFICACIÓN & Artículos susceptibles de cumplir con los criterios de búsqueda \\
\hline CRIBADO & 82 & 73 & 101 & 10 \\
\hline SELECCIÓN & $\mathrm{N}=0$ & $\mathrm{~N}=18$ & $\mathrm{~N}=2$ & $\mathrm{~N}=1$ \\
\hline & & & & \\
\hline TOTAL & 21 & & & \\
\hline
\end{tabular}

Fuente: elaboración propia 


\section{RESULTADOS}

Tabla 4.

Características de los artículos seleccionados

\begin{tabular}{|c|c|c|c|c|c|c|}
\hline Autor, año y país & Objetivos & Muestra & & $\begin{array}{l}\text { Tipo de estudio y } \\
\text { diseño de la } \\
\text { intervención }\end{array}$ & $\begin{array}{l}\text { Instrumentos de } \\
\text { medida }\end{array}$ & Resultados \\
\hline $\begin{array}{l}\text { Aidinopoulou, V., } \\
\text { y Sampson, D. G. } \\
\text { (2017). } \\
\text { Grecia }\end{array}$ & $\begin{array}{l}\text { Analizar } \\
\text { implementació } \\
\mathrm{n} \text { del modelo } \\
\text { mixto } \\
\text { learning) } \\
\text { Flipped } \\
\text { Classroom (FC) }\end{array}$ & $\begin{array}{l}\mathrm{N}=49 \\
11 \text { años }\end{array}$ & & $\begin{array}{l}\text { Diseño cuasi- } \\
\text { experimental } \\
\text { Grupo } \\
\text { experimental } \\
\text { (FC) } \\
\text { Grupo control } \\
\text { (no FC) }\end{array}$ & 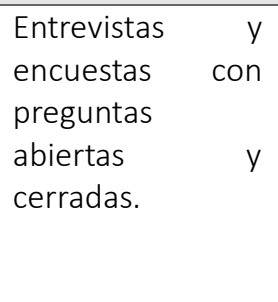 & $\begin{array}{l}\text { Uso más efectivo } \\
\text { del tiempo. } \\
\text { Menos impacto } \\
\text { en puntajes de } \\
\text { memorización en } \\
\text { los } 2 \text { grupos. }\end{array}$ \\
\hline $\begin{array}{l}\text { Amro, F., y } \\
\text { Borup, J. (2019) } \\
\text { Whashington }\end{array}$ & $\begin{array}{l}\text { Descubrir la } \\
\text { percepción y } \\
\text { experiencia de } \\
\text { los maestros en } \\
\text { la } \\
\text { implementació } \\
n \text { del modelo } \\
\text { mixto. }\end{array}$ & $\begin{array}{l}\mathrm{N}=11 \\
\text { Maestros }\end{array}$ & & $\begin{array}{l}\text { Estudio de caso } \\
\text { cualitativo }\end{array}$ & $\begin{array}{l}\text { Entrevistas } \\
\text { semiestructurada } \\
\mathrm{s}\end{array}$ & $\begin{array}{l}\text { Reconocimiento } \\
\text { de la dificultad en } \\
\text { la } \\
\text { personalización } \\
\text { del aprendizaje; } \\
\text { falta de tiempo } \\
\text { en el desarrollo } \\
\text { profesional; falta } \\
\text { de acceso a } \\
\text { recursos. }\end{array}$ \\
\hline $\begin{array}{l}\text { Akgündüz, } \\
\text { y Akınoğlu, O. } \\
\text { (2017). } \\
\text { Turquia }\end{array}$ & $\begin{array}{l}\text { Valorar el impacto } \\
\text { en los resultados } \\
\text { académicos y } \\
\text { motivacional en la } \\
\text { implementación } \\
\text { del modelo mixto. } \\
\text { (FC) }\end{array}$ & $\begin{array}{l}\mathrm{N}=74 \\
12-13 \text { años }\end{array}$ & & $\begin{array}{l}\text { Grupo de } \\
\text { control (Gc), } \\
\text { Grupo } \\
\text { experimental } 1 \\
\text { (Ge1) Grupo } \\
\text { experimental } 2 \\
\text { (Ge2+redes } \\
\text { sociales) }\end{array}$ & $\begin{array}{l}\text { Recopilación de } \\
\text { datos } \\
\text { cuantitativos; } \\
\text { escala de Likert. } \\
\text { Recopilación de } \\
\text { datos cualitativos; } \\
\text { entrevista } \\
\text { semiestructurada } \\
\text {. }\end{array}$ & $\begin{array}{lr}\text { Aumento } & \text { en } \\
\text { resultados } & \\
\text { académicos } & \text { y } \\
\text { motivacional en } \\
\text { grupo } \\
\text { experimental } \\
\text { (Ge1), no en } \\
\text { grupo } \\
\text { experimental } \\
\text { reforzado } \\
\text { redes (Ge2) }\end{array}$ \\
\hline $\begin{array}{lll}\text { Banjer, } & \text { F., } & \text { y } \\
\text { Zagami, } & & \text { J. } \\
\text { (2016) } & & \\
\text { Australia } & & \\
& & \\
& & \\
& & \end{array}$ & $\begin{array}{l}\text { Mejorar } \\
\text { comunicación } \\
\text { familia-escuela } \\
\text { en entornos de } \\
\text { enseñanza } \\
\text { combinada. }\end{array}$ & $\begin{array}{l}\text { Estudiantes, } \\
\text { docentes } \\
\text { familias } \\
\text { centro } \\
\text { educativo. }\end{array}$ & $\begin{array}{r}y \\
\text { del }\end{array}$ & $\begin{array}{l}\text { Diseño de } \\
\text { investigación } \\
\text { por encuesta. }\end{array}$ & $\begin{array}{l}\text { Encuestas } \\
\text { Entrevistas } \\
\text { personales } \\
\text { Cuestionarios }\end{array}$ & $\begin{array}{l}\text { Canales de } \\
\text { comunicación } \\
\text { mejorados } \\
\text { pudiendo dar } \\
\text { mejor respuesta } \\
\text { y apoyo al } \\
\text { alumnado. }\end{array}$ \\
\hline $\begin{array}{l}\text { Ceylan, V. K., y } \\
\text { Kesici, A. } \quad \text { E. } \\
\text { (2017). } \\
\text { Turquia }\end{array}$ & $\begin{array}{l}\text { Valorar } \\
\text { resultados de la } \\
\text { efectividad del } \\
\text { aprendizaje } \\
\text { combinado. }\end{array}$ & $\begin{array}{l}\mathrm{N}=53 \\
11 \text { años }\end{array}$ & & $\begin{array}{l}\text { Diseño cuasi- } \\
\text { experimental } \\
\text { con pre-test y } \\
\text { pos-test. Grupo } \\
\text { control y Grupo } \\
\text { experimental } \\
\text { (FC) }\end{array}$ & $\begin{array}{l}\text { Prueba t de } \\
\text { muestras } \\
\text { independientes. } \\
\text { ANOVA } \\
\text { SPSS } 21\end{array}$ & $\begin{array}{l}\text { Se descubrió } \\
\text { que el grupo } \\
\text { experimental } \\
\text { que ha } \\
\text { estudiado en un } \\
\text { entorno de } \\
\text { aprendizaje } \\
\text { mixto } \\
\text { académicament } \\
\text { e más exitoso. }\end{array}$ \\
\hline
\end{tabular}




\begin{tabular}{|c|c|c|c|c|c|}
\hline Autor, año y país & Objetivos & Muestra & $\begin{array}{c}\text { Tipo de estudio y } \\
\text { diseño de la } \\
\text { intervención }\end{array}$ & $\begin{array}{l}\text { Instrumentos de } \\
\text { medida }\end{array}$ & Resultados \\
\hline $\begin{array}{l}\text { Çetinkaya, M. } \\
\text { (2017) } \\
\text { Turquia }\end{array}$ & $\begin{array}{l}\text { Valorar efecto } \\
\text { sobre el } \\
\text { rendimiento } \\
\text { académico en } \\
\text { entorno de } \\
\text { aprendizaje } \\
\text { mixto. (FC) }\end{array}$ & $\begin{array}{l}\mathrm{N}=74 \\
12 \text { años }\end{array}$ & $\begin{array}{l}\text { Diseño cuasi- } \\
\text { experimental } \\
\text { con pre-test y } \\
\text { pos-test. Grupo } \\
\text { control y Grupo } \\
\text { experimental } \\
\text { (FC) }\end{array}$ & $\begin{array}{l}\text { Autoevaluación. } \\
\text { Cuestionario }\end{array}$ & $\begin{array}{l}\text { Diferencias } \\
\text { estadísticas } \\
\text { significativas para } \\
\text { el Grupo } \\
\text { experimental. }\end{array}$ \\
\hline $\begin{array}{l}\text { Çırak Kurt, S., y } \\
\text { Yıldırım, } \\
\text { (2018) } \\
\text { Turquia }\end{array}$ & $\begin{array}{l}\text { Determinar las } \\
\text { percepciones } \\
\text { del alumnado } \\
\text { en entrono } \\
\text { combinado }\end{array}$ & $\begin{array}{l}\mathrm{N}=31 \\
\text { 9-10 años. }\end{array}$ & $\begin{array}{l}\text { Este estudio se } \\
\text { realizó con el } \\
\text { método Q } \\
\text { para descubrir } \\
\text { cómo los } \\
\text { estudiantes } \\
\text { percibían el } \\
\text { aprendizaje } \\
\text { combinad. }\end{array}$ & $\begin{array}{l}\text { Datos } \\
\text { cuantitativos: } \\
\text { Formulario (18 } \\
\text { items). } \\
\text { Encuestas con } \\
\text { preguntas } \\
\text { abiertas que } \\
\text { complementan } \\
\text { los datos } \\
\text { cuantitativos. }\end{array}$ & $\begin{array}{l}\text { Importancia del } \\
\text { docente en su } \\
\text { organización y } \\
\text { preparación de las } \\
\text { clases. } \\
\text { Responsabilidad } \\
\text { del alumno y } \\
\text { calidad de los } \\
\text { materiales. }\end{array}$ \\
\hline $\begin{array}{l}\text { D’addato, T., y } \\
\text { Miller, L. R. R. } \\
\text { (2016) } \\
\text { California }\end{array}$ & $\begin{array}{l}\text { Analizar efecto } \\
\text { sobre el } \\
\text { rendimiento } \\
\text { académico en } \\
\text { entorno de } \\
\text { aprendizaje } \\
\text { mixto. (FC) }\end{array}$ & $\begin{array}{l}\mathrm{N}=27 \\
8-11 \text { años }\end{array}$ & $\begin{array}{l}\text { Periodos: } \\
\text { Trimestre 1; no } \\
\text { implementación } \\
\text {. Trimestre 2; } \\
\text { implementación } \\
\text { parcial. } \\
\text { Trimestre 3; } \\
\text { implementación } \\
\text { completa. }\end{array}$ & $\begin{array}{l}10-15 \quad \text { min. } \\
\text { Iniciales, } \\
\text { cuestionario } \\
\text { sobre contenido } \\
\text { visionado en } \\
\text { casa.. }\end{array}$ & $\begin{array}{l}\text { La enseñanza } \\
\text { mixta, fomenta la } \\
\text { motivación y } \\
\text { confianza en el } \\
\text { alumnado. }\end{array}$ \\
\hline $\begin{array}{l}\text { Dey, P., y } \\
\text { Bandyopadhya } \\
\text { y, S. (2019) } \\
\text { India }\end{array}$ & $\begin{array}{l}\text { ldentificar } \\
\text { factores } \\
\text { influyentes en } \\
\text { la calidad } \\
\text { educativa a } \\
\text { través del } \\
\text { aprendizaje } \\
\text { combinado. }\end{array}$ & $\begin{array}{l}\mathrm{N}=228 \\
79 \text { discentes } \\
\text { de } 3 \text { escuelas } \\
\text { de familias } \\
\text { desfavorecidas } \\
\end{array}$ & $\begin{array}{l}90 \text { días } \\
\text { mediante el } \\
\text { aprendizaje } \\
\text { combinado. } \\
\text { Comparación en } \\
\text { el puntaje de } \\
\text { bien estar y } \\
\text { aprendizaje. }\end{array}$ & $\begin{array}{l}\text { Medición } \\
\text { teniendo en } \\
\text { cuenta las } \\
\text { variables } \\
\text { independientes: } \\
\text { nivel } \\
\text { socioeconómico, } \\
\text { apoyo familiar y } \\
\text { entorno escolar. }\end{array}$ & 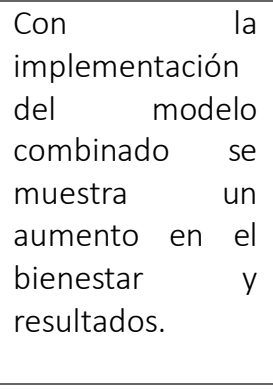 \\
\hline $\begin{array}{ll}\text { Fazal, } & \text { M., y } \\
\text { Bryant, } & \text { M. } \\
\text { (2019) } & \\
\text { Texas } & \end{array}$ & $\begin{array}{l}\text { Analizar efecto } \\
\text { sobre el } \\
\text { rendimiento } \\
\text { académico en } \\
\text { entorno de } \\
\text { aprendizaje } \\
\text { mixto (FC) }\end{array}$ & $\begin{array}{l}\mathrm{N}=812 \\
12 \text { años }\end{array}$ & $\begin{array}{l}\text { Diseño cuasi- } \\
\text { experimental } \\
\text { Grupo } \\
\text { experimental } \\
\text { (FC) } \\
\text { Grupo control } \\
\text { (no FC) }\end{array}$ & $\begin{array}{l}\text { Prueba T de dos } \\
\text { muestras. } \\
\text { Comparación de } \\
\text { puntajes de } \\
\text { enseñanza mixta } \\
\text { y no mixta.. }\end{array}$ & $\begin{array}{l}\text { Diferencia } \\
\text { estadística } \\
\text { significativa para } \\
\text { el grupo } \\
\text { experimental. }\end{array}$ \\
\hline $\begin{array}{lll}\text { Girmen, P., } & \text { y } \\
\text { Kaya, M. } & \text { F. } \\
\text { (2019) } & & \\
\text { Turquia } & & \end{array}$ & $\begin{array}{l}\text { Descubrir el } \\
\text { enriquecimient } \\
\text { o del desarrollo } \\
\text { de habilidades } \\
\text { básicas a través } \\
\text { del modelo } \\
\text { mixto (FC). }\end{array}$ & $\begin{array}{l}\text { N=23 } \\
10 \text { años } \\
\text { Familias }\end{array}$ & $\begin{array}{l}\text { Actividades } \\
\text { basadas en el } \\
\text { juego a través } \\
\text { del modelo } \\
\text { mixto. }\end{array}$ & $\begin{array}{l}\text { Realización de } \\
\text { actividades } \\
\text { basadas en el } \\
\text { juego. Resolución } \\
\text { de problemas y } \\
\text { posibles } \\
\text { deficiencias. }\end{array}$ & $\begin{array}{l}\text { Alumnado y } \\
\text { Familias } \\
\text { valoraron } \\
\text { positivamente el } \\
\text { modelo. }\end{array}$ \\
\hline $\begin{array}{l}\text { Hwang, G. J., y } \\
\text { Lai, C. L. (2017) } \\
\text { Grecia }\end{array}$ & $\begin{array}{ll}\text { Valorar } & \text { el } \\
\text { enfoque } & \\
\text { interactivo } & \text { de } \\
\text { libros } & \\
\end{array}$ & $\begin{array}{l}\mathrm{N}=45 \\
10 \text { años. } \\
2 \text { aulas }\end{array}$ & $\begin{array}{l}\text { Diseño cuasi- } \\
\text { experimental: } \\
\text { Grupo } \\
\text { experimental }\end{array}$ & $\begin{array}{l}\text { Prueba pre-test y } \\
\text { post-tes. } \\
\text { Cuestionario de } \\
\text { autoeficacia. }\end{array}$ & $\begin{array}{l}\text { Mostraron } \\
\text { resultados más } \\
\text { beneficiosos el } \\
\text { grupo }\end{array}$ \\
\hline
\end{tabular}




\begin{tabular}{|c|c|c|c|c|c|}
\hline Autor, año y país & Objetivos & Muestra & $\begin{array}{c}\text { Tipo de estudio y } \\
\text { diseño de la } \\
\text { intervención }\end{array}$ & $\begin{array}{l}\text { Instrumentos de } \\
\text { medida }\end{array}$ & Resultados \\
\hline & $\begin{array}{l}\text { electrónicos y } \\
\text { modelo mixto } \\
(\mathrm{FC}) \text {. }\end{array}$ & & $\begin{array}{l}\text { (FC+libro } \\
\text { electrónico) } \\
\text { Grupo control } \\
\text { (FC) }\end{array}$ & & experimental. \\
\hline $\begin{array}{l}\text { Khader, N. S. K. } \\
\text { (2016) } \\
\text { Jordania }\end{array}$ & $\begin{array}{l}\text { Comprobar la } \\
\text { eficacia del } \\
\text { aprendizaje } \\
\text { combinado. }\end{array}$ & $\begin{array}{l}\mathrm{N}=108 \\
9 \text { años }\end{array}$ & $\begin{array}{l}\text { Diseño cuasi- } \\
\text { experimental: } \\
\text { Grupo } \\
\text { experimental } \\
\text { (FC) } \\
\text { Grupo control } \\
\text { (no FC) }\end{array}$ & $\begin{array}{l}\text { Comparación } \\
\text { estadística entre } \\
\text { medias de las } \\
\text { calificaciones en } \\
\text { las unidades } \\
\text { didácticas. }\end{array}$ & $\begin{array}{l}\text { Existe una } \\
\text { diferencia } \\
\text { significativa entre } \\
\text { las medias, } \\
\text { siendo superior la } \\
\text { del Grupo } \\
\text { experimental. }\end{array}$ \\
\hline $\begin{array}{l}\text { Macaruso, P., } \\
\text { Wilkes, S., } \\
\text { Franzén, S., y } \\
\text { Schechter, R. } \\
\text { (2019) } \\
\text { Massachusetts }\end{array}$ & $\begin{array}{l}\text { Valorar la } \\
\text { eficacia del } \\
\text { modelo } \\
\text { combinado en } \\
\text { aprendizaje de } \\
\text { lectura en } \\
\text { alumnado de } \\
\text { bajos niveles } \\
\text { SES (similar } \\
\text { CAES) }\end{array}$ & $N=83$ & $\begin{array}{l}\text { Uso del } \\
\text { programa } \\
\text { Core5. } \\
63 \text { alumnos } \\
\text { continuaron con } \\
\text { el programa } \\
\text { durante } 2 \text { cursos } \\
\text { más. }\end{array}$ & $\begin{array}{l}\text { LexiaVR Core5 } \\
\text { VR Lectura. } \\
\text { ANOVA para ver } \\
\text { las diferencias de } \\
\text { puntuación. }\end{array}$ & $\begin{array}{lr}\begin{array}{l}\text { Beneficios } \\
\text { alumnado }\end{array} & \text { en } \\
\text { utilizó } & \text { el } \\
\text { programa } & \\
\text { incluso a largo } \\
\text { plazo. }\end{array}$ \\
\hline $\begin{array}{l}\text { Martínez, S. G. } \\
\text { (2016) } \\
\text { España }\end{array}$ & $\begin{array}{l}\text { Valoración del } \\
\text { alumnado sobre } \\
\text { la modalidad } \\
\text { mixta. }\end{array}$ & $\begin{array}{l}\mathrm{N}=7044 \\
7-15 \text { años. }\end{array}$ & $\begin{array}{l}\text { Plataforma } \\
\text { Moodle10 } \\
\text { Alojamiento de } \\
70 \text { cursos. }\end{array}$ & $\begin{array}{l}\text { Cuestionario ad } \\
\text { hoc. }\end{array}$ & $\begin{array}{l}\text { Los resultados } \\
\text { mostraron que } \\
\text { un } 81,5 \% \\
\text { requiere ayuda, } \\
\text { apoyo, } \\
\text { colaboración del } \\
\text { adulto de forma } \\
\text { puntual } \\
\text { continua. }\end{array}$ \\
\hline $\begin{array}{l}\text { Mora Reyes, J. } \\
\text { Z., y Morales } \\
\text { Rivera, S. P. } \\
\text { (2016) } \\
\text { Colombia }\end{array}$ & $\begin{array}{l}\text { Mejorar la } \\
\text { competencia } \\
\text { lecto-escritora } \\
\text { a través del } \\
\text { actividades } \\
\text { enriquecidas } \\
\text { con b- } \\
\text { learning. }\end{array}$ & $\begin{array}{l}\mathrm{N}=9 \\
5-6 \text { años }\end{array}$ & $\begin{array}{l}\text { Realización de } \\
\text { 3-4 actividades } \\
\text { semanales con } \\
\text { apoyo } \\
\text { tecnológico y } \\
\text { colaboración de } \\
\text { la familia. }\end{array}$ & $\begin{array}{l}\text { Observación } \\
\text { directa. } \\
\text { Entrevistas. } \\
\text { Encuesta familia }\end{array}$ & $\begin{array}{l}\text { La } \\
\text { implementación } \\
\text { fortaleció los } \\
\text { procesos de } \\
\text { lectoescritura del } \\
\text { alumnado. }\end{array}$ \\
\hline $\begin{array}{l}\text { Prescott, J. E., } \\
\text { Bundschuh, K., } \\
\text { Kazakoff, E. R., } \\
\text { y Macaruso, P. } \\
\text { (2018) } \\
\text { EE.UU }\end{array}$ & $\begin{array}{l}\text { Comprobar la } \\
\text { eficacia del } \\
\text { modelo } \\
\text { combinado. } \\
\text { Alumnado de } \\
\text { bajos niveles } \\
\text { SES (CAES) }\end{array}$ & $\begin{array}{l}\mathrm{N}=722 \\
\text { Hasta } 11 \text { años }\end{array}$ & $\begin{array}{l}\text { Instrucción } \\
\text { ELA Lexia } \\
\text { Reading } \\
\text { Core5 } \\
\text { Prueba pre- } \\
\text { test y post- } \\
\text { test. }\end{array}$ & $\begin{array}{l}\text { ANOVA Medidas } \\
\text { repetidas. } \\
\text { Diferencia en } \\
\text { pruebas pre-test y } \\
\text { post-test. }\end{array}$ & $\begin{array}{l}\text { Diferencia } \\
\text { positivas } \\
\text { significativas del } \\
\text { alumnado que } \\
\text { participó en el } \\
\text { aprendizaje } \\
\text { combinado. }\end{array}$ \\
\hline $\begin{array}{l}\text { Schechter, R. L., } \\
\text { Kazakoff, E. R., } \\
\text { Bundschuh, K., } \\
\text { Prescott, J. E., y } \\
\text { Macaruso, P. } \\
\text { (2017) } \\
\text { EEUU }\end{array}$ & $\begin{array}{l}\text { Descubrir el } \\
\text { Papel/Rol del } \\
\text { docente en el } \\
\text { entorno } \\
\text { combinado. }\end{array}$ & $\begin{array}{l}\mathrm{N}=19366 \\
624 \text { escuelas } \\
\text { Hasta } 10 \text { años }\end{array}$ & $\begin{array}{l}\text { Utilización del } \\
\text { programa } \\
\text { Core5 } \\
\text { Medición de } \\
\text { habilidades } \\
\text { lectoras. }\end{array}$ & $\begin{array}{l}\text { Comparación de } \\
\text { aulas con entorno } \\
\text { combinado y } \\
\text { aulas sin entorno } \\
\text { combinado. }\end{array}$ & $\begin{array}{l}\text { Mejoras } \\
\text { significativas en } \\
\text { habilidades } \\
\text { lectoras en las } \\
\text { aulas } \\
\text { entorno con } \\
\text { combinado }\end{array}$ \\
\hline
\end{tabular}




\begin{tabular}{|c|c|c|c|c|c|}
\hline Autor, año y país & Objetivos & Muestra & $\begin{array}{l}\text { Tipo de estudio y } \\
\text { diseño de la } \\
\text { intervención }\end{array}$ & $\begin{array}{l}\text { Instrumentos de } \\
\text { medida }\end{array}$ & Resultados \\
\hline (Providence) & & & & & $\begin{array}{l}\text { según rol del } \\
\text { docente. }\end{array}$ \\
\hline
\end{tabular}

\begin{tabular}{|c|c|c|c|c|c|}
\hline $\begin{array}{l}\text { Seage, S. J. y } \\
\text { Türegün, M. } \\
\text { (2020). } \\
\text { EEUU } \\
\text { (Florida) }\end{array}$ & $\begin{array}{lr}\text { Valorar los } \\
\text { efectos en el } \\
\text { aprendizaje }\end{array}$ & $\begin{array}{l}\mathrm{N}=129 \\
\text { 9-11 años }\end{array}$ & $\begin{array}{l}\text { Diseño cuasi- } \\
\text { experimental: } \\
\text { Grupo } \\
\text { experimental } \\
\text { Grupo control de } \\
\text { Modelo } \\
\text { instrucción 5E } \\
\text { (Participación, } \\
\text { Exploración, } \\
\text { Explicación, } \\
\text { Elaboración y } \\
\text { Evaluación) }\end{array}$ & $\begin{array}{l}\text { Plataforma Core } \\
\text { Team } \\
\text { MANOVA de } 2 \\
\text { grupos. }\end{array}$ & $\begin{array}{l}\text { Resultados } \\
\text { estadísticos } \\
\text { significativament } \\
\text { e más altos en el } \\
\text { entorno } \\
\text { combinado. } \\
\text { Alumnado de } \\
\text { bajos niveles } \\
\text { socioeconómicos } \\
\text { tienen a alcanzar } \\
\text { niveles más altos } \\
\text { en estos } \\
\text { entornos. }\end{array}$ \\
\hline $\begin{array}{lr}\text { Song, Y., } \quad \text { Y } \\
\text { Kapur, } & \text { M. } \\
\text { (2017). } & \\
\text { Hong Kong } & \end{array}$ & $\begin{array}{l}\text { Comparar } \\
\text { diseño } \\
\text { pedagógico en } \\
\text { aula invertida } \\
\text { con el aula } \\
\text { invertida con } \\
\text { diseño de } \\
\text { fracaso } \\
\text { productivo. }\end{array}$ & $\begin{array}{l}\mathrm{N}=50 \\
11-12 \text { años } \\
2 \text { aulas }\end{array}$ & $\begin{array}{l}\text { Estudio cuasi- } \\
\text { experimental. } \\
\text { Comparación de } \\
\text { diseños para } \\
\text { una unidad } \\
\text { didáctica de } 2 \\
\text { semanas con los } \\
\text { dos tipos de FC. }\end{array}$ & $\begin{array}{lr}\text { Fase } & \text { I: } \\
\text { Actividades } & \text { de } \\
\text { resolución } & \text { de } \\
\text { problemas } & \text { en } \\
\text { términos } & \text { de; } \\
\text { involucrarse, } & \\
\text { explorar } & \text { y } \\
\text { explicar. } & \\
\text { Fase } & \text { II: } \\
\text { Consolidación del } \\
\text { concepto. }\end{array}$ & $\begin{array}{l}\text { Ambas clases } \\
\text { tuvieron una } \\
\text { mejora } \\
\text { significativa a } \\
\text { nivel } \\
\text { procedimental } \\
\text { pero a nivel } \\
\text { conceptual } \\
\text { mostró mejores } \\
\text { resultados el aula } \\
\text { invertida } \\
\text { tradicional. }\end{array}$ \\
\hline $\begin{array}{l}\text { Yaghmour, K. S. } \\
\text { (2016) } \\
\text { Jordania }\end{array}$ & $\begin{array}{ll}\text { Valorar } & \text { la } \\
\text { efectividad } & \text { en } \\
\text { el modelo de } & \text { de } \\
\text { enseñanza } & \\
\text { combinado. }\end{array}$ & $\begin{array}{l}N=97 \\
9 \text { años }\end{array}$ & $\begin{array}{l}\text { Método cuasi- } \\
\text { experimental. } \\
\text { Grupo } \\
\text { experimental } \\
\text { (enseñanza } \\
\text { combinada) } \\
\text { Grupo de } \\
\text { control } \\
\text { (enseñanza no } \\
\text { combinada) }\end{array}$ & $\begin{array}{l}\text { Cuestionarios al } \\
\text { grupo de control y } \\
\text { experimental. } \\
\text { ANCOVA } \\
\text { bidireccional parar } \\
\text { las medias de los } \\
\text { dos grupos. }\end{array}$ & $\begin{array}{l}\text { Diferencias } \\
\text { estadísticas } \\
\text { significativament } \\
\text { e superiores en el } \\
\text { desempeño del } \\
\text { alumnado del } \\
\text { grupo } \\
\text { experimental. }\end{array}$ \\
\hline
\end{tabular}




\section{DISCUSIÓN}

El análisis de contenido de los 21 artículos científicos que componen este estudio permite establecer los principales elementos distintivos de las investigaciones realizadas en este campo, según los criterios seleccionados.

Se destaca el creciente número de investigaciones centradas en la etapa de Educación Primaria, lo que refleja el aumento del interés en los últimos tiempos, encontrando que la mayor parte de las publicaciones realizadas en este quinquenio se concentra en el año 2017 (42\%). Y, en relación al país de origen de cada uno de los trabajos consultados, los datos muestran que el modelo de aula invertida es objeto de reciente implementación en el sistema escolar y de análisis y reflexión en distintas partes del mundo. Resaltan Estados Unidos y Turquía por ser los países con más artículos publicados atendiendo a los filtros de búsqueda.

Los objetivos y pretensiones de los artículos seleccionados han sido en su gran mayoría sobre la eficacia del modelo de b-learning (77\% de los artículos) y el resto sobre la percepción del alumnado y del profesorado. En cuanto a los diseños metodológicos, la mayoría de las investigaciones han optado por utilizar el método cuasi-experimental; estudios de medidas repetidas con grupo experimental y grupo control, y con pruebas de pre-test y post-test.

Las aportaciones e investigaciones sobre el b-learning en el aprendizaje del alumnado de Educación Primaria, se ve reflejada en los resultados de los distintos estudios analizados. Así señalamos que, tanto Çırak y Yıldırım (2018) como Amro y Borup (2019), en el desarrollo e implementación del modelo, además de las valoraciones positivas, resaltan la importancia del docente como la clave para el éxito. En el estudio de Dey y Bandyopadhyay (2019) se valora positivamente los beneficios en la utilización del modelo de b-learning, aunque advierten de los riesgos que puede producir la brecha digital. Y Seage y Türegün (2020), y Macaruso, et al. (2019) apuntan hacia una mejora significativa en alumnado con ambiente desfavorecido en la implantación del modelo b-learning, consideran que hay una mejora de rendimiento gracias a este modelo.

En el estudio de Yaghmour (2016) se reconoce la eficacia del modelo, pero incluye algunos factores asociados que ayudan e influyen en dicha eficacia, por ejemplo: la individualización del aprendizaje, el aprendizaje basado en el alumnado, el autodescubrimiento y otras tendencias innovadoras en la enseñanza. También Aidinopoulou y Sampson, (2017) en sus resultados resaltan la eficacia del modelo en cuanto al uso efectivo del tiempo en el proceso educativo. $Y$ D'addato y Miller (2016) junto a Akgündüz y Akınoğlu (2016) reconocen la eficacia del modelo destacando especialmente el fomento de actitudes como el entusiasmo, la confianza o la motivación. En esa misma línea Martínez (2016) acentúan la importancia de la autonomía dentro del proceso de implementación de b-learning y Song y Kapur (2017) comparan el modelo b-learning de aula invertida con el aula invertida en el fracaso productivo, concediendo a este último mayor eficacia.

Del análisis de estos estudios se concluye que sobre las intervenciones basadas en el modelo blearning para la etapa de Educación Primaria, en su gran mayoría, se les ha realizado una valoración positiva en cuanto a su eficacia. Sin embargo, en el modelo b-learning están implicados muchos factores (Yaghmour, 2016) y su eficacia dependerá de su nivel de 
participación. De estos factores implicados, que muchos autores dan como implícitos en el modelo, apuntamos el aprendizaje cooperativo y el aprendizaje activo.

Finalmente, señalar que los estudios sobre b-learning en Educación Primaria son escasos y posiblemente aumentarán debido al crecimiento en la implantación del modelo. También se prevé que el modelo amplíe su ratio de impacto y es muy posible que se extrapole a otros campos educativos como educación de adultos, educación social... Lo que se evidencia, sin ningún género de dudas, es que el sistema educativo está experimentando un momento de adaptación importante y será fundamental seguir investigando sobre la introducción de la educación b-learning en el sistema educativo. Sin embargo, como comentábamos anteriormente, la mayoría de los estudios analizados están enfocados hacia la efectividad del modelo en cuanto a los resultados académicos. A lo que agregamos que sería positivo consensuar un cambio de tendencia en este sentido y orientar los estudios hacia otros horizontes. En ese sentido cabe destacar los estudios de Seage y Türegün (2020) y Macaruso, Wilkes, Franzén y Schechter (2019), ya que enfocaron el estudio hacia el alumnado con niveles socioeconómicos desfavorecidos. También sería destacable el estudio de D’addato y Miller (2016), junto al de Akgündüz y Akınoğlu (2016) porque, además de la eficacia del modelo, resaltan el fomento de actitudes que produce.

\section{CONCLUSIONES}

Como muestra la Tabla 4, dentro de la modalidad b-learning, uno de los modelos más representativos es el Flipped classroom, modalidad donde el enriquecimiento del proceso educativo se produce gracias a las dinámicas que se producen en el aula. Lamentablemente, como muestra García (2020), este modelo también se ha visto muy perjudicado en esta época pandémica ya que es imposible generar esa interacción y cooperativismo en el aula. Es cierto que en las aulas está llegando el modelo b-learning con carácter urgente y también por necesidad debido a las circunstancias provocadas por la pandemia, no por intereses de mejora o calidad educativa. Pero sería importante e interesante que el modelo se fuera instaurando en el sistema educativo como una alternativa más para el enriquecimiento del proceso educativo del alumnado.

En los artículos analizados, la mayoría buscan el mejoramiento académico y unos pocos centran su atención de forma directa o indirecta al fomento de otras actitudes o mejoras del proceso educativo. Pero ninguno de los artículos analizados ha puesta la atención al potencial que puede tener la gestión del aula a través del modelo mixto. En este modelo se distingue por plantear dos escenarios educativos, uno presencial y el otro no presencial, mediándose éste último por las herramientas tecnologías actuales. Esto significa que habrá un espacio virtual en el proceso educativo y es aquí donde debemos poner el foco a las nuevas posibilidades que se pueden generar. Rodríguez (2008), nos habla de e-herramientas, e-actividades, y tecnoalumnado donde será el docente el que tendrá que conjugar estos elementos para que el proceso se vea enriquecido.

En este contexto, se debería plantear y valorar seriamente el potencial de la educación mixta. Es decir, hasta qué punto podríamos dar respuestas desde la modalidad no presencial a las demandas y retos de la modalidad presencial. En cualquier situación de aprendizaje es fundamental el aspecto social e interactivo, sobre todo en los niveles de Educación Primaria. 
¿Qué ocurre cuando no disponemos de ese factor social e interactivo presencial? ¿Qué consecuencias y efectos podrá provocar esa no presencialidad en el proceso de aprendizaje? Son preguntas que podemos plantearnos ante la situación que nos encontramos.

Es cierto que las tecnologías tienen un poder no solo para la comunicación sino también para la relación entre personas. A nivel educativo, se podría aprovechar este poder para interceder en algunos aspectos interpersonales. En las aulas podemos encontrar alumnado que esté más desplazado del grupo y esto puede afectar negativamente a dicho alumnado, por lo que sería necesario replantearse y reflexionar sobre las posibilidades que nos brindan la tecnología para poder facilitar su socialización. Debemos plantearnos cómo desde la virtualidad podemos enlazar, interceder, aproximar, facilitar, acercar, generar... actividades y contextos que ayuden a esa socialización.

Si bien las Tecnologías de la Información y Comunicación (TIC) han supuesto cambios decisivos en nuestras prácticas educativas habituales, personales y colectivas y que resulta un fenómeno que difícilmente puede detenerse, resulta necesaria una profunda reflexión acerca del impacto que tiene en la manera en que nos relacionamos a través de ellas y por ende aprendemos. En palabras de Adell y Castañeda (2010), hablar de educación y TIC hoy ya no implica únicamente "integrar" las TIC en un proceso educativo formal y estandarizado. Actualmente las TIC son el entorno en el que se producen muchas de las interacciones y la comunicación que son la base del aprendizaje permanente de las personas

En esas interacciones y comunicación que se producen en el proceso educativo es necesario humanizar el uso de las tecnologías. El concepto humanización, como acto de humanizar, se ve interpelado por innumerables factores y variables, aunque con un denominador común que considera al ser humano como centro, integrando la capacidad de relación con otros. De esta forma, la utilización de TIC no se puede elevar sin más a la categoría de ámbito de aprendizaje propiamente dicho, ni por supuesto implica un fin en sí mismo dentro de los procesos de aprendizaje (Díaz y Laurian 2013). Por tanto, antes de comenzar una tarea humanizadora de la TIC en el ámbito de la educación, es preciso tener siempre presente cómo aprende el ser humano, tanto a nivel individual como en la colectividad. Se trata de poder definir estrategias que aporten elementos valiosos para el aprendizaje mediante el uso adecuado y pertinente de los recursos tecnológicos que nos ofrecen las TIC. Implica distanciarse de su carácter más instrumental para extraer y potenciar su faceta más humana.

De la misma manera, los docentes deberán fomentar y reestructurar nuevos contextos donde todo el alumnado se sienta integrado, prestando especial atención al desplazado o rechazado. Es decir, plantearse cómo mediante el uso de las tecnologías y una metodología correcta podemos conseguir un ambiente integrador.

Estas últimas aportaciones, explicitadas en el párrafo anterior, sirven para reflexionar sobre el enfoque recurrente y mayoritario de los artículos analizados y exigen nuevos planteamientos sobre este tema que cuestionen la funcionalidad del modelo. En concreto, cuanto antes son necesarias respuestas a interrogantes de este nivel: ¿qué aportaciones procura el modelo blearning para el crecimiento personal de cada alumno y alumna?, ¿y para el grupo-clase? ¿Cómo se consigue mejorar la socialización del alumnado a través del modelo b-learning?, y, para concluir, ¿̇cómo se puede gestionar la "socialización virtual" en esta situación excepcional en la que se encuentra una sociedad intensamente digitalizada, aunque sumida en la perplejidad? 


\section{RECONOCIMIENTO}

Los resultados ofrecidos forman parte del proyecto de tesis doctoral: "Diseño, implementación y valoración del modelo aula invertida en la etapa de Educación Primaria". Investigación realizada en el marco del Programa de Doctorado en Educación (Código 3117. RD 99/2011) de la Facultat de Filosofia i Ciències de l’Educació de la Universitat de València.

\section{REFERENCIAS}

Adell, J., y Area M. (2009). eLearning: Enseñar y aprender en espacios virtuales. En J. De Pablos (Coord.), Tecnología Educativa. La formación del profesorado en la era de Internet (pp. 391- 424). Aljibe. http://bit.ly/3vMN30N

Adell, J., y Castañeda, L. J. (2010). Los Entornos Personales de Aprendizaje (PLEs): una nueva manera de entender el aprendizaje. En R. Roig Vila y M. Fiorucci, Claves para la investigación en innovación y calidad educativas. La integración de las Tecnologías de la Información y la Comunicación y la Interculturalidad en las aulas. Stumenti di ricerca per l'innovaziones e la qualità in ámbito educativo. La Tecnologie dell'informazione e della Comunicaziones e l'interculturalità nella scuola (19-30). Marfil - Roma TRE Universita degli studi http://bit.ly/20Rinas

Aidinopoulou, V. y Sampson, D. (2017). An Action Research Study from Implementing the Flipped Classroom Model in Primary School History Teaching and Learning. Educational Technology \& Society, 20(1), 237-247. https://bit.ly/393JHfN

Akgunduz, D. y Akinoglu, O. (2016). The Effect of Blended Learning and Social Media-Supported Learning on the Students' Attitude and Self-Directed Learning Skills in Science Education. Turkish Online Journal of Educational Technology-TOJET, 15(2), 106-115. https://bit.ly/2Pfvg1k

Amro, F., y Borup, J. (2019). Exploring Blended Teacher Roles and Obstacles to Success When Using Personalized Learning Software. Journal of Online Learning Research, 5(3), 229-250. https://bit.ly/3caQtIW

Banjer, F., y Zagami, J. (2016). Is the School Experience for Early Phase Students, Their Parents, and Teachers Improved through the Use of Managed Online Tools? Australian Association for Research in Education. AARE Conference 2016 Melbourne, Victoria. https://bit.ly/3IEXQW6

Bartolomé, A. (2004). Blended Learning. Conceptos básicos. Pixel-Bit, Revista de Medios y Educación, 23, 7-20. https://bit.ly/2PjMVVA

Çetinkaya, M. (2017). Designing and Applying Web Assisted Activities to Be Used in Flipped Classroom Model. International Journal of Evaluation and Research in Education, 6(2), 128-137. https://bit.ly/3IJQReh 
Ceylan, V. K., y Elitok Kesici, A. (2017). Effect of blended learning to academic achievement. Journal of Human Sciences, 14(1), 308-320. https://www.jhumansciences.com/ojs/index.php/IJHS/article/view/4141

D'addato, T., y Miller, L. R. (2016). An inquiry into flipped learning in fourth grade math instruction. The Canadian Journal of Action Research, 17(2), 33-55. http://bit.ly/316FjZk

Dey, P., y Bandyopadhyay, S. (2019). Blended learning to improve quality of primary education among underprivileged school children in India. Education and Information Technologies, 24(3), 1995-2016. https://doi.org/10.1007/s10639-018-9832-1

Díaz, J. L. R., y Laurian, J. (2013). Humanización del aprendizaje en la era de la información: una arista andragógica. Revista Electrónica" Actualidades Investigativas en Educación", 13(3), 1-18. https://bit.ly/3lla4gt

Elliot, J. (1997). La investigación-acción en educación. Morata.

Fazal, M., y Bryant, M. (2019). Blended learning in middle school math: The question of effectiveness. Journal of Online Learning Research, 5(1), 49-64. https://bit.ly/3reSQIE

Girmen, P., y Kaya, M. (2019). Using the Flipped Classroom Model in the Development of Basic Language Skills and Enriching Activities: Digital Stories and Games. International Journal of Instruction, 12(1), 555-572. https://bit.ly/2OZF1ku

Hwang, G. J., y Lai, C. L. (2017). Facilitating and bridging out-of-class and in-class learning: An interactive e-book-based flipped learning approach for math courses. Journal of Educational Technology \& Society, 20(1), 184-197. https://bit.ly/3sgDpkP

Khader, N. S. K. (2016). The Effectiveness of Blended Learning in Improving Students' Achievement in Third Grade's Science in Bani Kenana. Journal of Education and Practice, 7(35), 109-116. https://bit.ly/3ccTSRb

Llorente, M. (2008). Actitudes de los alumnos universi-tarios en procesos de formación blended learning. Revista Internacional de Ciencias Sociales y Humanidades, XVIII, 2, 91-111. https://bit.ly/3f4oxIL

Macaruso, P., Wilkes, S., Franzén, S., y Schechter, R. (2019). Three-year longitudinal study: Impact of a blended learning program-Lexia ${ }^{\circledR}$ Core $5^{\circledR}$ Reading - on reading gains in lowSES kindergarteners. Computers in the Schools, 36(1), 2-18. https://doi.org/10.1080/07380569.2018.1558884

Martínez, S. G. (2016). Percepciones y valoraciones de alumnos en edad escolar sobre su experiencia de aprendizaje en un programa semipresencial de lengua y cultura españolas. RIED. Revista Iberoamericana de Educación a Distancia, 19(2), 237-262. http://dx.doi.org/10.5944/ried.19.2.15398

Morán, L. (2012). Blended-learning. Desafío y oportunidad para la educación actual. Edutec, Revista Electrónica de Tecnología Educativa, 39 (a188), 1-19. https://doi.org/10.21556/edutec.2012.39.371 
Palau, R., Gopal, J., Suñé, X., y Seritjol, F. (2015). Ventajas y desventajas de la aplicación del flipped classroom. Comunicación y Pedagogía, 285-286, 36-40.

Perez Gomez, A. I. (1998). La cultura escolar en la sociedad neoliberal. Morata.

Picciano, A. (2014). Introduction to Blended learning: research perspectives. En A. Picciano, C. R. Dziuban y C. R. Graham (Ed.), Blended learning: research perspectives. Routledge. https://doi.org/10.4324/9781315880310

Prescott, J. E., Bundschuh, K., Kazakoff, E. R., y Macaruso, P. (2018). Elementary school-wide implementation of a blended learning program for reading intervention. The Journal of Educational Research, 111(4), 497-506. https://doi.org/10.1080/00220671.2017.1302914

Reyes, J. Z. M., y Rivera, S. P. M. (2016). Fortalecimiento en los procesos lecto-escritos en primera infancia a través de blended-learning. REICE: Revista Iberoamericana sobre Calidad, Eficacia y Cambio en Educación, 14(1), 117-135. https://doi.org/10.15366/reice2016.14.1.007

Rodríguez, L. J. C. (2020). Efectos del coronavirus en el sistema de enseñanza: aumenta la desigualdad de oportunidades educativas en España. Revista de Sociología de la Educación-RASE, 13(2), 114-139. https://doi.org/10.7203/RASE.13.2.17125

Rodríguez, V. A. (2008). Tecnologías de la Información y la Comunicación, Sociedad y Educación. Tebar.

Román, F., Marín, D., y Peirats, J. (2017). Clase invertida e inclusión educativa: análisis del estado de la cuestión. Comunicación y Pedagogía, Revista de Educación y Tecnología, 297-298, 69-73.

Rosado, L. da Silva y Bohadana, E. (2007). Análise da ferramenta wiki para cooperação e colaboração no ambiente virtual de aprendizagem moodle. In E-Tic - Encontro de educação e tecnologias de informação e comunicação. Universidade Estácio de Sá, Rio de Janeiro, Brasil. https://doi.org/10.17851/1983-3652.4.1.55-67

Salinas, J. (1995): Organización escolar y redes: Los nuevos escenarios del aprendizaje. En Cabero, J. y Martínez. (Coord.), Nuevos canales de comunicación en la enseñanza (89117). Centro de Estudios Ramón Areces. https://www.researchgate.net/publication/263730298

Salinas, J. (2012). La investigación ante los desafíos de los escenarios de aprendizaje futuros. RED. Revista de Educación a Distancia, 32, 35-58. https://revistas.um.es/red/article/view/271251

Schechter, R. L., Kazakoff, E. R., Bundschuh, K., Prescott, J. E., y Macaruso, P. (2017). Exploring the impact of engaged teachers on implementation fidelity and reading skill gains in a blended learning reading program. Reading Psychology, 38(6), 553-579. http://dx.doi.org/10.1080/02702711.2017.1306602 
Seage, S. J., y Türegün, M. (2020). The effects of blended learning on STEM achievement of elementary school students. International Journal of Research in Education and Science (IJRES), 6(1), 133-140. https://doi.org/10.46328/ijres.v6i1.728

Song, Y., y Kapur, M. (2017). How to flip the classroom "productive failure or traditional flipped classroom" pedagogical design? Journal of Educational Technology \& Society, 20(1), 292305. https://bit.ly/2Qy2CsZ

Tayebinik, M., y Puteh, M. (2012). Blended Learning or E-learning? International Magazine on Advances in Computer Science and Telecommunications, 3(1), 103-110. http://doi.org/10.1016/j.iheduc.2012.12.001

Yaghmour, K. S. (2016). Effectiveness of Blended Teaching Strategy on the Achievement of Third Grade Students in Mathematics. Journal of Education and Practice, 7(5), 65-73. https://bit.ly/39az3nH

\section{Para citar este artículo:}

Román, F. E., Marín, D., y Peirats, J. (2021). Revisión bibliográfica y análisis sobre b-learning y la socialización del alumnado en educación primaria. Edutec. Revista Electrónica De Tecnología Educativa, (77), 136-151. https://doi.org/10.21556/edutec.2021.77.1957 